\title{
Double-Buffer Traffic Shaper Modelling for Multimedia Applications in Slow Speed Network
}

\author{
Oluwafolake Olayiwola, Damilola Oyewo, Ayodeji Oluwatope, \\ Adesola Aderounmu, and Rotimi Adagundo \\ Comnet Laboratory, Center of Excellence in Software \\ Engineering, Department of Computer Science and Engineering, \\ Obafemi Awolowo University, Ile-Ife, Nigeria
}

\author{
folakeolayiwola@yahoo.com, dammyoyewo@yahoo.com, \\ aoluwato@oauife.edu.ng, gaderoun@oauife.edu.ng, \\ eadagun@oauife.edu.ng
}

\begin{abstract}
Lack of end-to-end quality-of-service support, unbounded packet-data delay, poorly adapted transport protocol and slow-speed network are factors militating against the deployment of realtime video streaming across the Internet at slow-speed. This paper presents a Double-Buffer Traffic Shaper (DBTS) for rate adaption of real-time video transmission in a slow-speed network using Transmission Control Protocol (TCP) for data transportation. The scheme was tested in Matlab and it was found to reduce variable bit rate (VBR) traffic burstiness to its barest minimum and variability to almost nil.
\end{abstract}

Keywords: computer networks, multimedia communication, transport protocol, slow-speed network

\section{Introduction}

As the internet is experiencing rapid growth, Network-based multimedia applications such as video conferencing, video-on-demand, E-learning and Telemedicine has become a vital and increasing application on the Internet therefore attracting research activities. The lack of end-to-end quality-of-service (QoS) support in today's Internet has caused significant difficulties to the deployment of these applications (Lam et al., 2005). Therefore, the issue of QoS provisioning has become more essential than ever. QoS must support a bandwidth guaranteed transmission service, and provide an efficient communication path for transmitting data under acceptable delay, delay jitter, network throughput and error rate (JerYang \& ChengChou, 2003). Bandwidth management and traffic control have become more complicated. Network control must support differ-

Material published as part of this publication, either on-line or in print, is copyrighted by the Informing Science Institute. Permission to make digital or paper copy of part or all of these works for personal or classroom use is granted without fee provided that the copies are not made or distributed for profit or commercial advantage AND that copies 1) bear this notice in full and 2) give the full citation on the first page. It is permissible to abstract these works so long as credit is given. To copy in all other cases or to republish or to post on a server or to redistribute to lists requires specific permission and payment of a fee. Contact Publisher@InformingScience.org to request redistribution permission. ent types of traffic classes at their required QoS and must efficiently utilize the network resources (Liang, 2004). TCP is recently becoming most acceptable as the transport layer protocol used for real- time streaming instead of UDP because TCP is faster, efficient, more responsive to network conditions and also integrates flow and congestion control scheme which removes the need for loss recovery and flow control at the 
application layer (Xiong et al., 2010). Transmission of real-time VBR video applications typically requires high bandwidth and low delay(Xiong et al., 2010; Xiong et al., 2009; Lam et al., 2005; Liang, 2004; JerYang \& ChengChou, 2003; Won \& Shim, 2002). These requirements have been identified as major challenges to the deployment of multimedia applications across the Internet in sub-Saharan countries particularly Nigeria. The reason is not far-fetched from the fact that these networks are slow-speed networks characterised by low speed and high delay as observed on end-user devices. In South-West Nigeria for example where this study was carried out, it was discovered; from informal interviews conducted for users on the campus on the average data rate that at peak period 5-10kbps was the available/guaranteed data rate. This is a far-cry from the experiences of average Internet users in Europe whose user-end data rate can be as high as $500 \mathrm{Mbps}$ described in literature as fast speed networks.

The focus of this work is to develop a rate adaptive scheme capable of guaranteeing the transmission of real-time VBR video across the Internet over slow-network at minimal delay and loss rate. A Double-Buffer Traffic Shaper (DBTS) for real-time VBR video traffic was formulated using network of queues. The scheme was modeled as a death and birth rate process. The contribution of this article is the proposition of a rate adaptive scheme for VBR Video traffic over slow-speed networks. The output result shows that the proposed scheme can successfully remove uneven distribution of video frames. The rest of the paper is organized as follow: Section two discusses related works; section three gives the description of the proposed scheme - Double-Buffer Traffic Shaper (DBTS). Section four presents the results while conclusions are given in Section five.

\section{Related Works}

Many researchers have worked on Real Time Variable Bit Rate (RT-VBR) traffic mobility scheme. Dinesh et al presented a delay jitter control algorithm that guarantees delay jitter in a packet-switched wide-area network (Dinesh et al., 1991). İbrahim introduced Virtual Scheduling Algorithm (VSA) for traffic shaping and achieved Lower loss rate (Ibrahim, 2000). de Cuetos et al and Lam et al designed network-adaptive video applications using MPEG-4 Fine-Grained Scalable (FGS) to adapt the coding rate of the streaming video to the variations of the available bandwidth ( de Cuetos et al., 2002; Lam et al., 2005). Bajic et al propose an end-to end buffer management scheme for video flow for robust and efficient scalable video communication Congestion control and was able to reduce packet loss and produce high video quality (Bajic et al., 2003). Arne and Jirka, generates real rate adaptive MPEG-4 streaming traffic using the Shaped Variable Bit Rate (SVBR) for adjusting the sending rate quality of service (QoS) performance for VBR video traffic (Arne \& Jirka, 2007). Xiong et al., propose a video frame schedule scheme for rate adaptive real-time video transmission over TCP to reduce end-to-end delay in real-time video (Xiong et al., 2009; Xiong et al., 2010). All of the above schemes were found unsuitable for the slow speed network. The reason being that design parameters and assumptions mainly as it concerns a link's minimum bandwidth were set to $\geq 1 \mathrm{Mbps}$ which is far greater the minimum obtainable in a typical slow-speed network.

\section{The Proposed Scheme (DBTS) Description}

\section{Video Transmission over the Internet}

The first step in the transmission of video is compression of the raw video using encoding coderdecoder scheme (CODEC) such as H.263, H.264, etc at the sender-end. The compressed video is passed to the sender-buffer within the application layer, thereafter passed to the TCP senderbuffer wherein they are fragmented into TCP data packets. From within the TCP sender-buffer the fragmented video frames are moved into the network. The video frames are later received into a TCP receiver-buffer wherein they are defragmented and decompressed. The decompressed 
video frames are then sent into the receiver playout-buffer. Fig. 1 depicts the stage by stage transmission of video frames from the sender to the receiver across the internet.

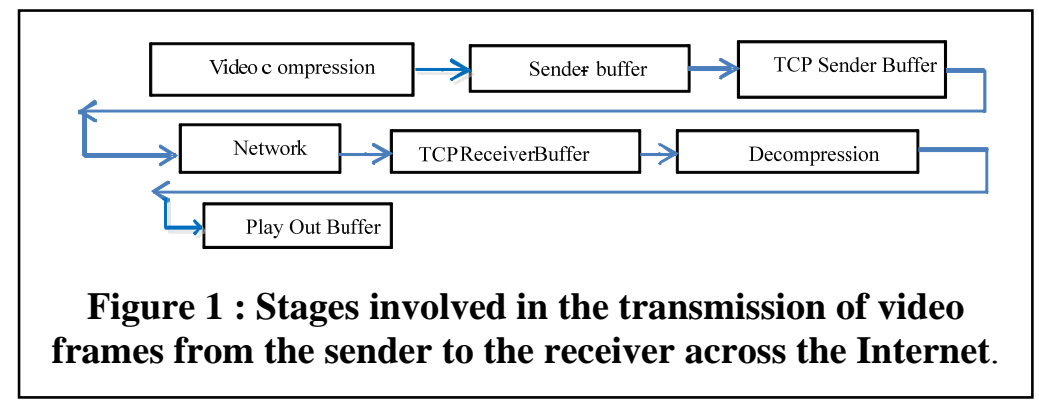

In real-time video transmission, packet delay is experienced as video frames proceed from one stage to another. The cumulation of these delays result into an end-to-end delay $\left(\mathrm{D}_{\mathrm{END}}\right)$. i.e

$$
\mathrm{D}_{\mathrm{END}}=\mathrm{D}_{\mathrm{VC}}+\mathrm{D}_{\mathrm{SB}}+\mathrm{D}_{\mathrm{TSB}}+\mathrm{D}_{\mathrm{N}}+\mathrm{D}_{\mathrm{TRB}}+\mathrm{D}_{\mathrm{D}}+\mathrm{D}_{\mathrm{P}}
$$

Where, $\mathrm{D}_{\mathrm{END}}$ - End-to-end delay; $\mathrm{D}_{\mathrm{VC}}$ - delay experienced during video compression;

$\mathrm{D}_{\mathrm{SB}}$ - delay experienced at sender- buffer; $\mathrm{D}_{\mathrm{N}}$ - delay experienced at network layer;

$\mathrm{D}_{\mathrm{TSB}}-$ delay experienced at TCP sender-buffer; $\mathrm{D}_{\mathrm{TBR}}-$ delay experienced at TCP receiverbuffer; $\mathrm{D}_{\mathrm{D}}-$ delay experienced during video decompression; $\mathrm{D}_{\mathrm{P}}-$ delay experienced at play out buffer.

Given that $\mathrm{D}_{\mathrm{VC}}, \mathrm{D}_{\mathrm{D}}, \mathrm{D}_{\mathrm{TRB}}$ and $\mathrm{D}_{\mathrm{P}}$ are commonly constant(Xiong et al.,2010), hence equation(1) becomes

$\mathrm{D}_{\mathrm{END}}=\mathrm{D}_{\mathrm{SB}}+\mathrm{D}_{\mathrm{TSB}}+\mathrm{D}_{\mathrm{N}}+\mathrm{C}$

The delay experienced at the application sender-buffer, TCP sender-buffer and in the network are variable and of major interest to researchers (Xiong et al., 2009; Xiong et al., 2010).

\section{The Double Buffer Traffic Shaper (DBTS)}

Due to the variability in $\mathrm{D}_{\mathrm{SB}}, \mathrm{D}_{\mathrm{TSB}}$, and $\mathrm{D}_{\mathrm{N}}$, and the possibility to curtail them, we wish to introduce a rate-adaptation scheme called DBTS. DBTS will be located between the application sender-buffer and the TCP sender buffer because bursty compressed video frames arriving from the application will be regulated by the scheme before arriving into the TCP sender-buffer. DBTS is a traffic shaping technique developed upon network of queues principle. The first queue as a $\mathrm{M} / \mathrm{D} / 1 / \mathrm{K}$ and the second queues as a $\mathrm{D} / \mathrm{D} / 1 / \mathrm{K}$. The flow of video frames is seen in fig. 2 . The arrival rate into the first buffer is markovian (Balsamo, 2010), its departure rate is deterministic at the feeding the second buffer, thereby ensuring that frame out flow from the second buffer is maintained at a constant rate. Video frames thereafter are sent into sender's TCP-buffer, hence achieving rate-adaptation. The frames flow dynamics can be expressed as a birth and death process. The transition is precipitated on H.263 encoder which sends an average of 10 frames per second. The arrival rate into the queue is Poisson, which implies that frames arrive in bursts (irregular spurts). The service rate is deterministic with mean departure rate, $\mu=\beta$ frames per second depending on the size of the queue. The inter-arrival rate in Figure 5 is inherited from the departure rate of the previous queue. There is a direct relationship between the two because the frame outflow of the $\mathrm{M} / \mathrm{D} / 1 / \mathrm{K}$ queue forms the inflow into the $\mathrm{D} / \mathrm{D} / 1 / \mathrm{K}$ queue. 


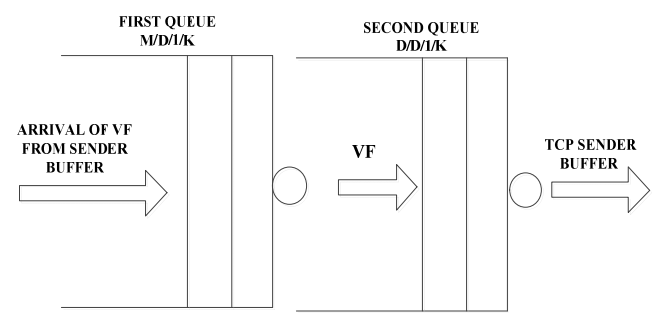

\section{Figure 2: Network of queues modeling of the proposed scheme}

Compressed video frames from the application layer sender buffer arrives into the first queue at higher rate but the rate at which the frames flow into the second queue are as follows:

Case 1: When current first queue size, a(t), approaches and above $75 \%$ capacity utilization, then current first queue outflow rate $\mathrm{r}_{\mathrm{y}}(\mathrm{t}+1)=\mathrm{r}_{\mathrm{y}}(\mathrm{t})^{*} \zeta$, where $\zeta$ belongs to the set of positive real numbers.

$$
\begin{aligned}
& \text { if }(a(t) \geq 0.75) \text { then } \\
& r_{y}(t+1)=r_{y}(t) * \zeta
\end{aligned}
$$

Case 2: When current first queue size, a $(\mathrm{t})$, operates below $25 \%$ capacity utilization, then current first queue outflow rate is halved i.e $\mathrm{r}_{\mathrm{y}}(\mathrm{t}+1)=\mathrm{r}_{\mathrm{y}}{ }^{*}$, such that $0.0>_{\gamma}<1.0$

Case 3: Otherwise, the flow rate is set at the default.

Assumptions:

Video frames inflow into First queue is markovian utilizing the H.263 Standard. Second queue size is assumed larger than the First queue i.e $(b>>a)$.

List of terms

$\mathrm{c}_{\mathrm{a}}$ - number of frames in first queue at time $\mathrm{t}$

$c_{b^{-}} \quad$ number of frames in second queue at time $t$

$r_{\mathrm{x}}$ : rate of Inflow to first queue

$r_{y}$ : rate of Inflow to second queue

$\mathrm{r}_{\mathrm{z}}$ : $\quad$ rate of Inflow to the TCP sender buffer

t: $\quad$ time(s)

a: $\quad$ size of First queue (number of video frames)

b: $\quad$ size of Second queue (number of video frames)

Input : uneven arrival of video frames, Output: even departure of video frames,

Initialization: $\mathrm{c}_{\mathrm{a}}, \mathrm{c}_{\mathrm{b}}, \mathrm{r}_{\mathrm{y}}, \mathrm{r}_{\mathrm{x}}$, out : array $[1, \ldots, \mathrm{n}]$.

$\mathrm{r}_{\mathrm{x}} \leftarrow \mathrm{H} .263$ trace data

begin

$$
\begin{aligned}
c_{a}(i+1)= & c_{a}(i)+r_{x}(i) ; \\
\operatorname{if}\left(c_{a}(i)>\right. & 0.75 * a) \\
& r_{y}(i+1)=r_{y}(i) * \zeta ;
\end{aligned}
$$




$$
\begin{aligned}
& \operatorname{elseif}\left(c_{a}(i)<0.25 * a\right) \\
& r_{y}(i+1)=r_{y}(i) * \gamma ;
\end{aligned}
$$

else

$$
r_{y}(i+1)=10 \text {; }
$$

end

$$
\begin{aligned}
& \operatorname{if}\left(\mathrm{c}_{\mathrm{a}}(\mathrm{i}+1)>0\right) \\
& \qquad \mathrm{c}_{\mathrm{a}}(\mathrm{i}+1)=\mathrm{c}_{\mathrm{a}}(\mathrm{i}+1)-\mathrm{r}_{\mathrm{y}}(\mathrm{i}+1) ;
\end{aligned}
$$

end

$$
\begin{aligned}
& \mathrm{c}_{\mathrm{b}}(\mathrm{i}+1)=\mathrm{c}_{\mathrm{b}}(\mathrm{i})+\mathrm{r}_{\mathrm{y}}(\mathrm{i}+1) ; \\
& \operatorname{if}\left(\mathrm{c}_{\mathrm{b}}(\mathrm{i}+1)>\mathrm{r}_{\mathrm{z}}\right) \\
& \quad \operatorname{out}(\mathrm{i})=\mathrm{r}_{\mathrm{z}} ; \\
& \quad \mathrm{c}_{\mathrm{b}}(\mathrm{i}+1)=\mathrm{c}_{\mathrm{b}}(\mathrm{i}+1)-\mathrm{r}_{\mathrm{z}} ;
\end{aligned}
$$

end

continue

\section{Results and Discussion}

The algorithm was implemented in Matlab using H.263 video trace file obtained from (http://trace.kom.aau.dk/trace/tut/html). The data file is a one-hour video session in H.263 format composed of a total of 25,432 frames. Arrival rates into the first queue was set to $r_{x} \in[1, \ldots, 15]$. At steady-state, we observed that the first queue size oscillates between 32-160 video frames with $\approx$ mean of 6 frames and variance 3.5 as depicted in Figure 5. Frame arrival rate into the first queue was markovian, with a mean value of $r_{x}=6$ and variance $=3.5$ as shown in Figure 3 , but the arrival rate into the second queue was found to be 6 and 1.4( mean and variance) for $\mathrm{r}_{\mathrm{y}}$ respectively as depicted in Figure 4 . The variability of the video data has been reduced by more $50 \%$. Also, we observed that the second queue size oscillates between 0-250 frames for about $90 \%$ of the simulation period and peaked at 370 as shown in Figure 6 . This result shows that the size of the second queue should be about twice the first queue for optimum performance. However, we are not unaware of the impact of mean packet delays which will be fully reported in subsequent papers.

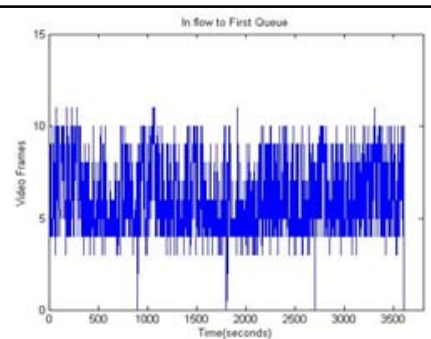

Figure 3: Frame distributions for $\mathbf{r}_{\mathbf{x}}$

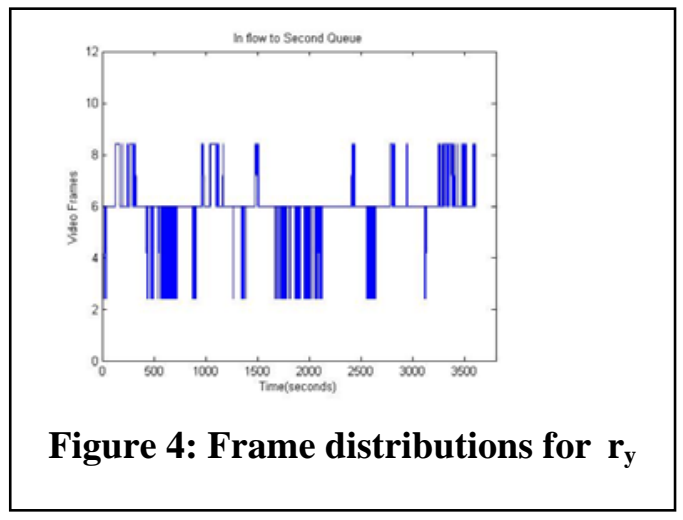




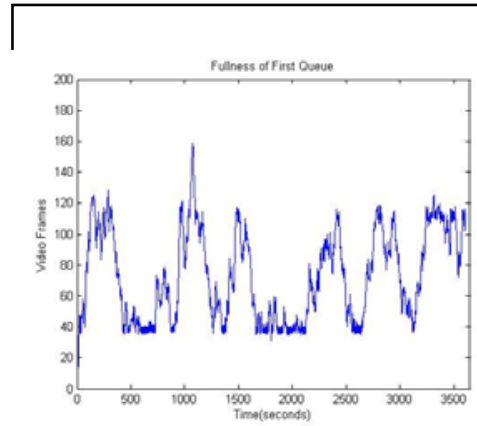

Figure 5: steady-state distribution for first queue size, $c_{a}$.

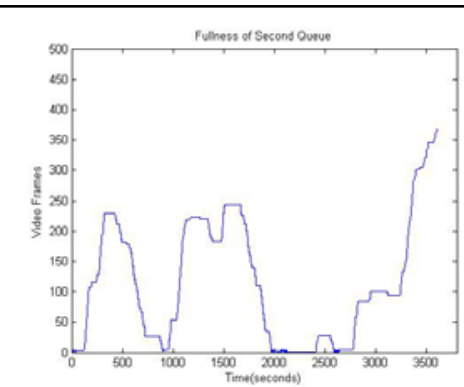

Figure 6: steady-state distribution of second queue size, $c_{b}$

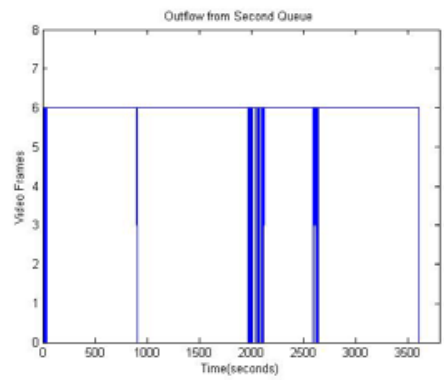

Figure 7: steady-state distribution of second queue outflow, $r_{z}$

The outflow of the first queue presents interesting results by curtailing the variability in the inflow into the second queue to a great extent. The simulation result gave a mean and variance for $r_{z}$ as 6 and 0 respectively as shown in Figure 7. These results confirmed our hypothesis.

\section{Conclusion}

In this paper, a Double-Buffer traffic shaper for rate adaption of real-time video transmission in a slow-speed network over TCP was presented. The proposed scheme attempts to eliminate the uneven distribution of video frames arrival from the application layer sender buffer and produces even distribution before the video frames are injected into the TCP sender buffer. For further work, the birth and death process will be analysed for steady-state dynamics. A packet-level modeling and simulation will be carried out in NS3 with the aim to carry out performance analysis of average packet delay, jitter and bit-error rate.

\section{References}

Arne, L., \& Jirka, K. (2007). Evalvid-RA: Trace driven simulation of rate adaptive MPEG-4 VBR video. Dept. of Communication Systems, Technical University of Berlin, Germany.

Bajic, I., OmeshTickoo, C., Balan, A., Kalyanaraman, S. \& Woods, J. (2003). Integrated end-to-end buffer management and congestion control for scalable video communications. Proceedings of IEEE Inter. Conf. on Image Processing, Barcelona, Spain, vol. 3, pp. 257-260.

Balsamo, S. (2010). A tool for the numerical solution of cooperating Markov chains in product-form, pp. $311-324$. 
de Cuetos, P., Guillote, P., Ross, K., \& Thoreau, D. (2002). Implementation of adaptive streaming of stored MPEG4 FGS video over TCP. Proceedings of IEEE Inter. Conf. on Multimedia and Expo, vol. 1, pp. 405-408.

Dinesh, C., Zhang, H., \& Ferrari, D. (1991). Delay jitter control for real-time communication in a packet switching network. Proceedings of TriComm. Retrieved from http://trace.kom.aau.dk/trace/tut $/ \mathrm{html}$

Doggen, J., Schueren, V., \& Filip. (2008). Design and simulation of a H.264 AVC video streaming model. Department of Applied Engineering, University College of Antwerp, Belgium.

Ibrahim, K. (2000). TCP traffic shaping in ATM networks. Faculty of Engineering and Architecture, Department of Electronics, Uludağ University, Görükle, Bursa-Turkey.

JerYang, S., \& ChengChou, H. (2003). Adaptive QoS parameters approach to modeling Internet performance. International Journal of Network Management, 13, 69-82.

Lam, L., Jack, Y., Lee, B., Liew, S., \& Wang, W. (2005). A transparent rate adaptation algorithm for streaming video over the internet. Department of Information Engineering, The Chinese University of Hong Kong, Shatin, N.T., Hong Kong.

Liang, Y. (2004). Real-time VBR video traffic prediction for dynamic bandwidth allocation. IEEE Transactions on Systems, Man, and Cybernetics, 34(1).

Won, Y., \& Shim, B. (2002). Empirical study of VBR traffic smoothing in wireless environment. Division of Electrical and Computer Engineering Hanyang University, Seoul, Korea.

Xiong, Y., Wu, M., \& Jia, W. (2009). Efficient frame schedule scheme for real-time video transmission across the internet using TCP. Journal of Networks,4(3), 216-223.

Xiong, Y., Wu, M., \& Jia, W. (2010). Delay prediction for real-time video adaptive transmission over TCP. Journal of ultimedia, 5(3), 216-222.

\section{Biographies}

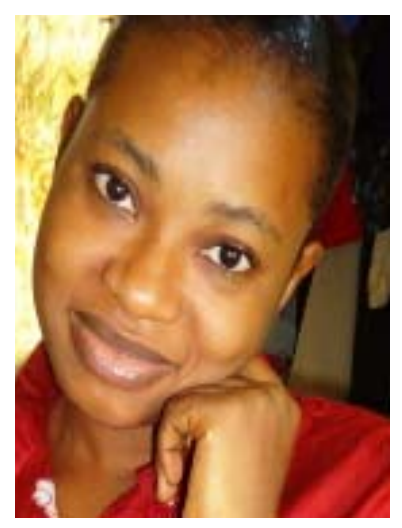

Folake Olayiwola is a M.Sc by research student of Computer Science in Department of Computer Science and Engineering, Obafemi Awolowo University, Ile-Ife Nigeria. She has a BSc. Degree in Computer Science from Olabisi Onabanjo University, Ago-Iwoye, Ogun-State, Nigeria. She is currently concluding her research work. Her research interest is in computer communication-QoS and protocol modeling and simulation

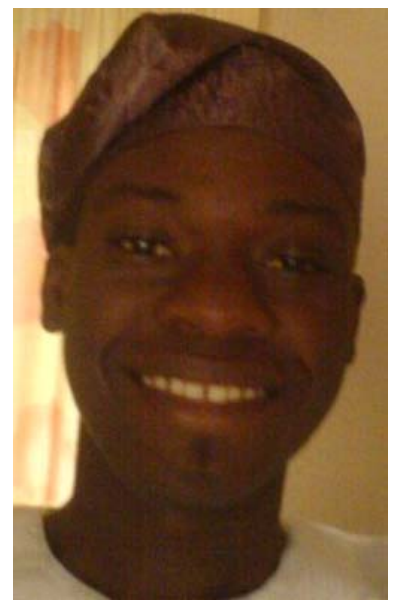

Damilola Oyewo is a M.Sc by research student of of Computer Science in Department of Computer Science and Engineering, Obafemi Awolowo University, Ile-Ife Nigeria. He has a BTech. degree in Computer Science from Ladoke Akintola University of Technology, Ogbomosho, Oyo-State, Nigeria. He is currently concluding his research work. His research interest is in computer communication-QoS and mathematical modeling of protocol. 


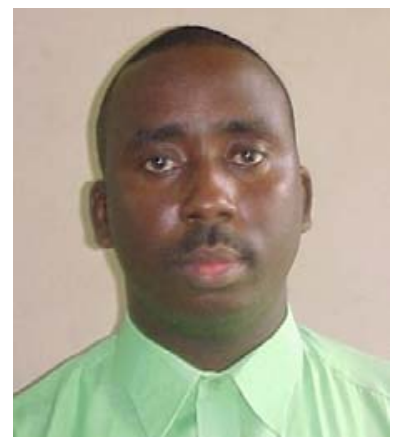

Ayodeji Oluwatope obtained BSc (Computer Engineering) '95, MSc (Computer Science) '03 and Ph.D (Computer Science) '08 all from the Obafemi Awolowo University, Ile-Ife, Nigeria. He is a Senior Lecturer in the Department of Computer Science and Engineering, Obafemi Awolowo University, Ile-Ife where he teaches and conducts research. $\mathrm{He}$ is the Leader of Network Utility Maximisation Sub-group (Comnet Labs) in the Department wherein he supervises Masters and Doctoral students. He has published several articles in journals and international conferences. He is a member of the Nigerian Society of Engineers, Nigerian Computer Society and the Nigerian Young Academy. He serves on the editorial board of a journal and conference proceedings.

Adesola Aderounmu is a Professor of Computer Science and Engineering, Obafemi Awolowo University, Ile-Ife, Nigeria and head of the Comnet Laboratories (Comnet Labs). He currently teaches and conducts research in computer networks. He has graduated numerous Masters students and 6 Ph.D students in Computer Science. He has published widely in international journals of high repute and served as Editor-in-Chief of Nigerian Computer Society's International Journal of Computer Science and Its Applications.

Rotimi Adagunodo is a Professor and head of Computer Science and Engineering, Obafemi Awolowo University(OAU), Ile-Ife, Nigeria. He is currently the Director of the Step-BWorld/Bank designated Center of Excellence in Software Engineering, OAU., Ile-Ife. He currently teaches and conducts research in Distributed Systems and Software Engineering. He has graduated numerous Masters and Doctoral students in Computer Science. He has published widely in international journals of high repute and served as Chairman of the International Conference on the Application of ICT to Teaching, Research, and Administration, AICTTRA. 